\title{
PREVALÊNCIA DE BACTÉRIAS MULTIRRESISTENTES EM UMA UNIDADE DE TERAPIA INTENSIVA
}

\author{
PREVALENCE OF MULTIRESISTENT BACTERIA IN AN INTENSIVE \\ CARE UNIT
}

\author{
Larissa Queiroz de Oliveira ${ }^{1}$ \\ Rosângela Vidal de Negreiros ${ }^{2}$ \\ Cristiana Barbosa da Silva Gomes ${ }^{3}$ \\ Hélio Lopes da Silveira ${ }^{4}$ \\ Andréia Oliveira Barros Sousa
}

\begin{abstract}
RESUMO: Esta pesquisa tem como objetivo identificar a prevalência de bactérias multirresistentes em uma Unidade de Terapia Intensiva (UTI). Trata-se de um estudo descritivo, epidemiológico, retrospectivo e transversal de abordagem quantitativa, tendo por base os dados previamente coletados pela Comissão de Controle de Infecção Hospitalar $(\mathrm{CCIH})$ nas fichas de notificação das Infecções Hospitalares entre 2017 a 2018, os dados foram digitados e processados no Pacote Estatístico para as Ciências Sociais (SPSS), versão 22.0, aplicou-se o Teste de Associação Qui-quadrado, bem como o Teste de Associação de Fisher. Na UTI do hospital estudado, o perfil dos pacientes diagnosticados com infecção hospitalar foram com idade maior ou igual a 60 anos $(59,1 \%)$, havendo equilíbrio entre os sexos masculino $(49,5 \%)$ e feminino $(50,5 \%)$. As fontes de infecção mais frequentes foram por SVD e CVC, ambos com taxas de $90,3 \%$ e a bactéria considerada mais prevalente foi a Klebsiella pneumoniae $(20,4 \%)$. Ainda, constatou-se o maior uso do antibiótico ciprofloxacino $(52,7 \%)$. Dessa forma, a implementação de programas intensivos de prevenção e controle de IRAS consiste numa abordagem eficiente para o controle da disseminação da resistência microbiana.
\end{abstract}

PALAVRAS CHAVE: Farmacorresistência Bacteriana; Transmissão de doença infecciosa do profissional para o paciente; Unidade de Terapia Intensiva.

\footnotetext{
1 Enfermeira Graduada pela Universidade Federal de Campina Grande-UFCG. E-mail: larissaq12.lq@gmail.com.

2 Professora orientadora. Doutoranda da USP. Mestre em Enfermagem. Docente do curso de Enfermagem do CCBS/UFCG. E-mail: negreiros.vidal@hotmail.com.

3 Enfermeira Graduada pela Universidade Federal de Campina Grande-UFCG. E-mail: redentor.cristiana@gmail.com.

${ }_{4}^{4}$ Mestrando da UEPB. Especialista em Enfermagem. Enfermeiro do setor de Epidemiologia do HUAC. E-mail: hlsil@hotmail.com.
} 
ABSTRACT: This research has as its goal the identification of multiresistant bacteria's prevalence in an Intensive care units (ICU). It is a descriptive, epidemiological, retrospective and transversal study with a quantitative approach, based on previously collected data from the hospital's infection control committee, through infection notification sheets filled between 2017 and 2018. The data was typed and processed in the Statistical Package for the Social Sciences (SPSS), version 22.0. A chi-square test was applied, as well as Fisher's exact test. In the ICU of the studied hospital, the diagnosed patients' profile with nosocomial infection was age greater than or equal to 60 years old (59.1\%), with balance between females (50.5\%) and males (49.5\%). The most prevalent infection sources were indwelling urinary catheters (IUC) and central venous catheters (CVC), both with rates of $90.3 \%$ and the most prevalent bacteria was Klebsiella pneumoniae (20.4\%). Still, it was found higher use of antibiotic ciprofloxacin (52.7\%). This way, the implementation of intensive prevention and control of health care-associated infections programs consists in an efficient approach to control bacterial resistance dissemination.

Descriptors: Drug Resistance Bacterial; Infectious Disease Transmission Professional-to- Patient; Intensive care unit. 


\section{INTRODUÇÃO}

As Unidades de Terapia Intensiva (UTI) constituem-se de estruturas complexas com monitorização contínua destinada ao atendimento de pacientes graves ou com descompensação de um ou mais sistemas orgânicos, e que necessitam de suporte e tratamento intensivo visando restabelecer a capacidade orgânica de recuperação. Geralmente, as internações são prolongadas e em uso de procedimentos invasivos, como: cateteres venosos centrais, intubações, sondas vesicais de demora e ventilação mecânica (PUGGINA et al., 2014).

Os índices de infecções permanecem elevados no Brasil, com maior incidência em hospitais de ensino ou universitários em comparação a outros hospitais, devido à variedade de doenças, à realização de procedimentos de alta complexidade, ao longo período de internação e ao contato de pacientes com diversos profissionais da saúde, incluindo os estudantes (PERNA et al., 2015).

Segundo a Agência Nacional de Vigilância Sanitária, a ocorrência de Infecções Relacionadas à Assistência à Saúde (IRAS) caracteriza-se como uma situação muito prevalecente nos serviços de saúde, levando-se ao uso das mais diversas classes de antimicrobianos em grandes proporções, favorecendo assim, a ocorrência de resistência microbiana, promovendo um grave problema de saúde pública em todo o mundo. (ANVISA, 2017a).

As IRAS representam uma das maiores ameaça à segurança do paciente, consideradas uma das fontes mais comuns de danos evitáveis. Destaca-se que, cerca de $20 \%$ a $30 \%$ dessas complicações infecciosas são preveníveis. Vale salientar que, o controle dessas infecções representa o caminho para melhorar a qualidade da assistência, reduzir custos e salvar vidas (SINÉSIO et al., 2018).

Na saúde pública, a resistência bacteriana representa um risco à qualidade de vida conquistada ao longo dos anos com o avanço da microbiologia, das engenharias, da farmácia e da medicina, comprometendo o orçamento dos sistemas 
de saúde sendo públicos ou privados, além de intensificar um problema de grande relevância quanto à prevalência das infecções hospitalares (COSTA, 2017).

A descoberta da penicilina por Alexander Fleming, em 1928, abriu o caminho a novos investimentos científicos no domínio da antibioticoterapia e, consequentemente à descoberta de novos antibióticos, propiciando o controle e a cura das doenças infecciosas (PEREIRA et al., 2005). No entanto, o desenvolvimento de pesquisas nesta área desencadeou a problemática quanto ao aparecimento de bactérias multirresistentes aos antibióticos. Alguns fatores contribuíram para o seu surgimento, como o uso indiscriminado de antibióticos, número limitado de recursos humanos, infecção cruzada direta ou indiretamente por microrganismos resistentes e vigilância epidemiológica deficiente. A circulação de bactérias multirresistentes nas UTI's vem dificultando o tratamento de algumas IRAS (GOMES et al., 2014).

A resistência microbiana é um problema de saúde pública associada a diversos fatores e fasciculado entre os sistemas de saúde de todo o mundo, segundo World Economic Forum Global Risks, a resistência bacteriana encontra-se listada como uma das grandes ameaças à saúde humana (BLAIR et al., 2015).

Tornando-se assim, relevante considerar de forma multidisciplinar, o maior número possível de estratégias de ação, contemplando desde a natureza das doenças infecciosas, os procedimentos farmacoterapêuticos existentes, os fatores ambientais, políticos e socioeconômicos pertinentes, visto que não existe solução definitiva para a resistência microbiana, em função da dinamicidade da evolução biológica dos organismos vivos (ANVISA, 2017a).

Diante dessa problemática, despertou o seguinte questionamento: Quais são as bactérias multirresistentes prevalentes em uma UTI de um hospital universitário?

A partir do exposto, evidencia-se a relevância da problemática sobre resistência bacteriana, devido ao crescente aumento da multirresistência à ação dos antibióticos, principalmente em UTI, dificultando a realização de uma terapêutica eficiente para infecções bacterianas. Dessa forma, esta pesquisa torna-se relevante por avaliar as bactérias multirresistentes mais prevalentes na UTI de um Hospital Universitário $(\mathrm{HU})$, identificando os perfis patogênicos, propondo estratégias para prevenção e redução dos índices de contaminações. 
Assim, este artigo tem como objetivos identificar a prevalência de bactérias multirresistentes em uma UTI de um HU; estabelecer a característica demográfica e clínica dos pacientes internados na UTI; descrever as prevalentes fontes de infecção; analisar os principais antibióticos utilizados de acordo com a cultura realizada; e propor estratégias para prevenção e redução dos índices de infecções por bactérias multirresistentes.

\section{PROCEDIMENTOS METODOLÓGICOS}

Trata-se de um estudo descritivo, epidemiológico, retrospectivo e transversal, de abordagem quantitativa, realizado na UTI de um HU, tendo por base os dados previamente coletados pela Comissão de Controle de Infecção Hospitalar $(\mathrm{CCIH})$ do Hospital nas fichas de notificação das Infecções Hospitalares, que foram preenchidas na UTI e arquivadas na sala da CCIH entre 2017 a 2018.

O procedimento de coleta de dados foi realizado através de busca ativa para análise das fichas arquivadas na sala da $\mathrm{CClH}$, o qual realizou-se um levantamento com 100 fichas de notificação, porém apenas 93 atenderam aos critérios de inclusão e exclusão, das quais apresentavam perguntas fechadas abordando as variáveis, como: sexo, idade, tempo de permanência no setor, diagnóstico inicial, tipo de infecção hospitalar, paciente colonizado por microrganismos resistentes durante a internação, uso de procedimentos invasivos e desfecho do tratamento dos pacientes. Para tanto, os pesquisadores fizeram visitas semanais ao Setor da CCIH.

Os dados foram organizados e distribuídos em planilha eletrônica no programa Microsolt Office Excel ${ }^{\circledR}$ e analisados utilizando o software Statistical Package for the Social Sciences (SPSS) versão 22.0. Aplicou-se o Teste de Associação Quiquadrado, bem como o Teste de Associação de Fisher. Nos casos que existiam associação entre as variáveis foi realizado a Razão de Chances (Odds Ratio). Estas análises foram efetivadas por meio do software R (R CORE TEAM, 2017).

O Teste Associação de Qui-quadrado verifica uma possível existência de associação entre variáveis. A hipótese nula desse teste é de não associação entre 
as variáveis, ou seja, são independentes. Enquanto, o Teste de Associação de Fisher representa significância na análise de contingência. Sobre a Razão de Chances, para Lee (1994) e Thompson et al. (1998), avalia- se a relação entre a chance de um paciente exposto possuir a condição de resistência, comparada à do paciente não exposto.

Esse estudo resulta do recorte do projeto intitulado "Gestão do risco de infecções relacionadas à assistência à saúde em Unidades de Terapia Intensiva de uma instituição pública na Paraíba", submetido ao Comitê de Ética em pesquisa do Hospital Universitário Alcides Carneiro, sendo aprovado sob CAAE: 92982418.2.0000.5182.

\section{ANÁLISE E DISCUSSÃO}

Os resultados enfatizam a prevalência de bactérias multirresistentes em uma UTI de um Hospital Universitário, merecendo destaque a grande quantidade de bactérias resistentes encontradas em amostras isoladas de pacientes diagnosticados com infecção hospitalar $(\mathrm{IH})$. Vale salientar que, as internações em UTI abrangem um pequeno subgrupo dos pacientes hospitalizados, representando 5 a $10 \%$ do total, com risco médio de infecção de 5 a 10 vezes maior do que outros setores e taxa de mortalidade de 10 a $80 \%$, de acordo com o perfil do paciente internado (PERNA et al., 2015).

$\mathrm{Na}$ Tabela 1, evidenciam-se as características demográficas e clínicas dos pacientes que desenvolveram IH durante a internação. Das pessoas acometidas por $\mathrm{IH}, 47(50,5 \%)$ eram do sexo feminino e 46 (49,5\%) eram masculinos, representando equilíbrio no acometimento das $\mathrm{IH}$ entre os sexos. Quanto a faixa etária de maior prevalência de $\mathrm{IH}$ ocorreu em pacientes com idade maior ou igual a 60 anos $(59,1 \%)$. Em idosos internados em UTI, as alterações das variáveis morfológicas e fisiológicas devem ser consideradas, pois repercutem nas diversas funções orgânicas, com destaque para a imunidade, tornando-os ainda mais vulneráveis a infecções, podendo causar um prolongamento da internação e no uso de 
procedimentos invasivos, aumentando os gastos com o tratamento e incrementando as chances de óbitos (SOUSA et al., 2017). Além disso, quanto ao desfecho hospitalar dos pacientes, constatou-se que 30,1\% evoluíram para o óbito.

Tabela 1 - Características demográficas e clínicas dos pacientes diagnosticados com IH em uma UTI de um Hospital publico da Paraíba $(n=93)$.

\begin{tabular}{lcc}
\hline Características & $\mathbf{n}$ & $\mathbf{\%}$ \\
\hline Sexo & 46 & 49,5 \\
\hline Masculino & 47 & 50,5 \\
\hline Feminino & & 40,9 \\
\hline Idade & 38 & 59.1 \\
\hline Menor que 60 anos & 55 & 4,3 \\
\hline Maior ou igual a 60 anos & 4 & 30,1 \\
\hline Desfecho & 28 & 1,1 \\
\hline Alta hospitalar & 1 & 64,5 \\
\hline Óbito & 60 &
\end{tabular}

$\mathrm{IH}$ : infecção hospitalar, UTI: unidade de terapia intensiva.

Fonte: Fichas arquivadas na $\mathrm{CCIH}, 2018$.

A tabela 2 apresenta os dispositivos invasivos como fontes de infecção mais prevalentes nos pacientes com IH, considerando-se a ventilação mecânica (VM), sonda nasogástrica (SNG), sonda nasoenteral (SNE), gastrostomia (GTT), traqueostomia (TQT), tubo orotraqueal (TOT), Sonda Vesical de Demora (SVD) e cateter venoso central (CVC).

Evidenciou-se a prevalência dos casos de infecção no trato urinário por SVD e infecção sistêmica por CVC, cujas taxas são semelhantes, correspondendo a 90,3\% dos casos. A infecção por SNG/SNE/GTT foi a segunda mais prevalente, perfazendo um total de $83,9 \%$, seguida de infecção no trato respiratório por VM, com $78,5 \%$. Os dispositivos invasivos que apresentaram menor prevalência de infecção foram TQT e TOT, com $37,6 \%$ e $19,4 \%$ dos casos, respectivamente.

A infecção de trato urinário (ITU) é uma das mais frequentes na população adulta, sendo caracterizada pela presença de agentes infecciosos e pela invasão do microrganismo para qualquer parte do trato urinário (ureter, bexiga e rins). Cerca de 
35 a $45 \%$ de todas as infecções hospitalares adquiridas são ITU, sendo que $80 \%$ estão relacionadas ao uso da SVD, procedimento invasivo comum em UTI (CARDOSO et al., 2014). Além disso, a contaminação pode ocorrer durante a inserção da sonda, resultante de falha na técnica asséptica; por via periuretral ascendente, por meio do ambiente extraluminal entre a sonda e o lúmen da uretra; e por contaminação intraluminal (ARAÚJO et al., 2018).

Os CVC são dispositivos rotineiramente implantados em pacientes críticos para auxiliar no tratamento e no cuidado. No entanto, constitui-se como um meio de disseminação de infecções locais ou sistêmicas, dependendo do tipo de cateter, técnicas de manipulação, frequência de manipulação além da suscetibilidade do indivíduo (ARAÚJO et al., 2018). As infecções do fluxo sanguíneo apresentam duas explicações comuns sobre a colonização. A primeira consiste na migração de microorganismos da pele para a incisão, local em que foi inserido o cateter, ocasionando colonização da ponta do cateter. A segunda resulta da contaminação do cateter, geralmente por meio de profissionais da assistência, motivando a colonização do mesmo por migração de microrganismos (BARROS et al., 2012).

Tabela 2 - Dispositivos invasivos como prevalentes fontes de infecção nos anos em uma Unidade de Terapia Intensiva de um Hospital Público da Paraíba.

\begin{tabular}{lcc}
\hline Dispositivos invasivos & $\mathbf{n}$ & $\mathbf{\%}$ \\
\hline SVD & 84 & 90,3 \\
\hline CVC & 84 & 90,3 \\
\hline SNG/SNE/GTT & 78 & 83,9 \\
\hline TOT & 18 & 19,4 \\
\hline TQT & 35 & 37,6 \\
\hline VM & 73 & 78,5 \\
\hline SVD: sonda & vesical de & demora, CVC: cateter \\
nasogástrica, SNE: sonda nasoenteral, GTT: gastrostomia, TOT: tubo orotraqueal,
\end{tabular}

Fonte: Fichas arquivadas na CCIH. HUAC, 2018.

$\mathrm{Na}$ tabela 3, o patógeno isolado com maior frequência nos pacientes diagnosticados com $\mathrm{IH}$ relatados neste estudo foi Klebisiela pneumoniae, com cerca de $20,4 \%$ dos casos. A Klebsiella pneumoniae é um bacilo gram-negativo que faz 
parte da microbiota intestinal, e sua virulência está associada à presença de uma cápsula polissacarídica, do sistema de captação de ferro, do fenótipo mucóide e do lipopolissarídeo tóxico. O aumento da mortalidade por infecções causadas por Klebsiella pneumoniae decorre principalmente de sepse, choque séptico, infecções da corrente sanguínea e da terapia antimicrobiana inadequada. A progressão da resistência de Klebsiella pneumoniae aos antibióticos tem causado grande preocupação desde a década de 1980 com o aparecimento da Klebsiella pneumoniae produtoras de Beta- lactamases de espectro estendido (PERNA et al., 2015).

Tabela 3 - Microrganismos isolados em pacientes diagnosticados com IH em uma UTI de um Hospital Universitário entre 2017 e 2018.

\begin{tabular}{lcc}
\hline Microrganismos isolados & $\mathbf{n}$ & $\mathbf{\%}$ \\
\hline Staphylococcus aureus & 5 & 5,4 \\
\hline Bgn (não fermentado) & - & - \\
\hline Pseudomonas aeruginosa & 14 & 15,1 \\
\hline Proteus sp & 7 & 7,5 \\
\hline Staphylococcus epidermidis & 4 & 4,3 \\
\hline Pseudomonas sp & 1 & 1,1 \\
\hline Escherichia coli & 1 & 1,1 \\
\hline Proteus mirabilis & 3 & 3,2 \\
\hline Klebsiella pneumoniae & 19 & 20,4 \\
\hline Acinetobacter baumannii & 6 & 6,5 \\
\hline
\end{tabular}

${ }^{*}$ Bgn: bacilo Gram negativo.

Fonte: Fichas arquivadas na $\mathrm{CCIH}, 2018$.

As principais bactérias multirresistentes responsáveis pelas IRAS nas UTI's e suas complicações são: Sthaphylococcus aureus, resistente à oxacilina, enterobactérias produtoras de beta lactamase de espectro estendido (ESBL), bactérias do grupo CESP (Citrobacter spp, Enterobacter spp, Serratia spp e Providencia spp), Enterococcus resistente à vancomicina, Klebsiella pneumoniae produtora de carbapenemase e Acinetobacter sp. (GOMES et al., 2014). Diante disso, o sistema de vigilância epidemiológica desempenha papel fundamental na detecção desses microrganismos, bem como na prevenção e controle. 
O combate à propagação de bactérias resistentes aos antimicrobianos e ao desenvolvimento de novos mecanismos de resistência requer uma abordagem conjunta de vários segmentos governamentais e da sociedade, além de envolver a necessidade de proposição de políticas que resultem em um amplo investimento em pesquisas, na aquisição de tecnologias e no desenvolvimento de recursos humanos (ANVISA, 2017a).

Além disso, a pesquisa apresenta ainda os principais antibióticos utilizados em uma UTI, dos quais evidenciou-se a partir da cultura realizada nos pacientes internados com diagnóstico de infecção hospitalar, um maior uso do quinolona ciprofloxacino com 52,7\%, seguido da cefalosporina ceftriaxona com $48,4 \%$ e do aminoglicosídeo gentamicina com 39,8\%. Enquanto, os antibióticos que apresentaram menor uso foram os glicopeptídeos vancomicina e teicoplanina, e a polimixina $B$, cujas taxas são semelhantes correspondendo a $1,1 \%$.

Os antimicrobianos representam a segunda classe de medicamentos mais utilizada em hospitais, é responsável por 20 a 50\% das despesas hospitalares com medicamentos, além de serem também prescritos em larga escala em nível ambulatorial. Este amplo uso pode afetar significativamente tanto a microbiota do indivíduo quanto a do ambiente hospitalar (ANVISA, 2017b).

Atualmente, as indústrias farmacêuticas carecem de alternativas terapêuticas contra bactérias patogênicas, devido aos mecanismos de multirresistência desenvolvidos nos diferentes antibióticos comercializados. Na última década, várias organizações como a Sociedade de Doenças Infecciosas da América (IDSA), os Centros de Controle e Prevenção de Doenças (CDC), a Organização Mundial da Saúde (OMS) e o Fórum Econômico Mundial (WEF), descreveram a resistência aos antibióticos como o foco nos relatos, por meio de conferências para acelerar a atividade de restrição e controle aos antimicrobianos (CÁRDENAS et al, 2019).

A resistência aos antibióticos é considerada um desperdício na economia, e para enfrentá- la é necessário investimento a longo prazo, de forma a fomentar o desenvolvimento de novos medicamentos, ferramentas de diagnóstico, vacinas e outras intervenções; fornecimento de suporte técnico-financeiro aos países em desenvolvimentos e fortalecimento dos sistemas de saúde, visando promover o acesso e a utilização eficaz de agentes antimicrobianos (OMS, 2014). 
Diante desse panorama, o Programa de Gerenciamento de Uso de Antimicrobianos envolve um conjunto de ações destinadas ao controle do uso desses medicamentos nos serviços de saúde, englobando desde o diagnóstico, a seleção, a prescrição e a dispensação adequadas, as boas práticas de diluição, conservação e administração, além da auditoria, monitoramento das prescrições, educação dos profissionais e pacientes, do monitoramento do programa até a adoção de medidas intervencionistas, assegurando resultados terapêuticos ótimos com mínimo risco potencial (ANVISA, 2017b).

Dessa forma, a interação da Comissão de Controle de Infecção Hospitalar e da equipe multiprofissional são de grande importância na realização de ações de prevenção e controle de infecções, estabelecendo critérios eficientes, como o monitoramento das prescrições médicas; controle do uso de antimicrobianos; realização de vigilância epidemiológica contínua; educação permanente com os funcionários (GASPAR et al., 2012).

\section{CONSIDERAÇÕES FINAIS}

Através da análise desse estudo, a microbiota hospitalar mais prevalentes na Unidade de Terapia Intensiva mostrou-se composta por 09 microorganismos, sendo que, a Klebsiella pneumoniae foi considerada a bactéria multirresistente mais prevalente na UTI do Hospital Universitário, com cerca de 20,4\% dos1010 casos. Além disso, notou-se predominância dos casos de ITU por SVD e infecção sistêmica por CVC, ambos correspondendo a 90,3\% dos casos. Enquanto as características dos pacientes diagnosticados com IH durante a internação, houve predomínio de pacientes com idade maior ou igual a 60 anos $(59,1 \%)$ e equilíbrio entre os sexos masculino $(49,5 \%)$ e feminino $(50,5 \%)$.

Ainda, constatou-se a partir da verificação dos antibiogramas, um maior uso do quinolona ciprofloxacino com $52,7 \%$, seguido da cefalosporina ceftriaxona, com $48,4 \%$ e do aminoglicosídeo gentamicina com 39,8\%. Enquanto os antibióticos que 
apresentaram menor uso foram os glicopeptídeos vancomicina e teicoplanina, e a polimixina $B$, cujas taxas são semelhantes correspondendo a $1,1 \%$.

Dessa forma, a implementação de programas intensivos de prevenção e controle de infecção relacionada à assistência à saúde consiste numa abordagem eficiente para o controle da disseminação da resistência microbiana no ambiente hospitalar. Componentes importantes desses programas incluem a vigilância, investigação e controle de surtos, protocolos de esterilização e desinfecção de equipamentos, além da implementação de práticas de cuidados ao paciente tais como higienização das mãos, isolamento e barreiras entre pacientes infectados/colonizados. Ademais, também inclui a necessidade de suporte de laboratório de microbiologia capaz de realizar identificação precoce da infecção, caracterização do perfil de resistência aos antimicrobianos auxiliando a tomada de decisão por parte dos profissionais de saúde (ANVISA, 2017a).

Diante disso, percebe-se a importância desse estudo, envolvendo a $\mathrm{CCIH}$ e a equipe multiprofissional, na realização de ações de prevenção e controle de infecções, estabelecendo critérios eficientes, orientando as atividades de educação continuada da instituição, voltadas, principalmente, para a capacitação dos profissionais e estudantes.

Além disso, sugere-se que estudos futuros sejam realizados no hospital, com a finalidade de analisar os indicadores, na perspectiva das $\mathrm{IH}$. E investir em conhecimentos sobre multirresistência bacteriana, devido às poucas publicações recentes disponíveis para o acesso abordando a temática. 


\section{REFERÊNCIAS BIBLIOGRÁFICAS}

Agência Nacional de Vigilância Sanitária. Plano Nacional para a Prevenção e o Controle da Resistência Microbiana nos Serviços de Saúde. Brasília, p. 1-84, maio, $2017 a$.

Agência Nacional de Vigilância Sanitária. Diretriz Nacional para Elaboração de Programa de Gerenciamento do Uso de Antimicrobianos em Serviços de Saúde. Brasília, p. 10, dezembro, 2017b.

ARAÚJO, Priscila Lopes. et al. Prevalencia de la infección relacionada con la asistencia a la salud en pacientes hospitalizados en unidad de cuidados intensivos. Enferm. glob. Murcia, v. 17 , n. 52, outubro, 2018.

ARNOLD, Ryan S. et al. Surgimento de bactérias produtoras de Klebsiella pneumoniae Carbapenemase (KPC). South Med J., v. 104, n. 1, p. 40-45, janeiro, 2011.

BARROS, Lívia Moreira. Prevalência de micro-organismo e sensibilidade antimicrobiana de infecções hospitalares em unidade de terapia intensiva de hospital público no Brasil. Rev. Ciênc. Farm. Básica Apl. Fortaleza, v. 33, n. 3, p. 429-435, 2012.

BLAIR, J. M. et al. Molecular Mechanisms of Antibiotic Resistance. Nature, v. 13, p. 42- 51, 2015.

CÁRDENAS, Lorena Liseth. et al. Nuevos antibióticos contra la resistencia bacteriana. Revista de la Asociación Colombiana de Infectología. Bogotá, v. 23, n. 4, p. 382-387, out- dez, 2019.

CARDOSO, Simone Aparecida Caetano. MAIA, Luiz Faustino dos Santos. Cateterismo vesical de demora na UTI adulto: o papel do enfermeiro na prevenção de infecção do trato urinário. Revista Científica de Enfermagem. São Paulo, v. 4, n. 12, p. 5-14, 2014.

COSTA, Anderson Luiz Pena da; JUNIOR, Antonio Carlos Souza Silva. Resistência bacteriana aos antibióticos e Saúde Pública: uma breve revisão de literatura. Revista Macapá, v.7, n. 2, p. 45-57, maio/ago. 2017.

GASPAR, Maria Dagmar da Rocha. et al. Prevalência de infecções hospitalares em um hospital geral de alta complexidade no município de Ponta Grossa. Acta Scientiarum. Maringá, v. 34, n. 1, p. 23-29, jan-jun, 2012

GOMES, Andreza Cristina. et al. Caracterização das infecções relacionadas à assistência à saúde em unidade de terapia intensiva. Revista de enfermagem UFPE online. Recife, v. $8, n$. 6, p. 1577-1585, junho, 2014.

LEE, James. Odds ratio or relative risk for cross-sectional data?. International Journal of Epidemiology, v.23, p. 201-203, fevereiro, 1994.

MOREIRA, Leila Beltrami. Princípios para o uso de antimicrobianos. Rev AMRIGS. Porto alegre, v. 48, n. 2, p. 73-152, 2004.

NOGUEIRA, Paula Sacha Frota. et al. Perfil da infecção hospitalar em um hospital universitário. Revista de Enfermagem. Rio de Janeiro, v. 17, n. 1, p. 96-101, jan-mar, 2009.

PEREIRA, Ana Leonor; PITA, João Rui. Alexander Fleming (1881-1955): Da descoberta da penicilina (1928) ao Prêmio Nobel (1945). Revista da Faculdade de Letras História. Porto, III Série, v. 6, p. 129-151, 2005. 
PERNA, Thaíssa Daulis Gonçalves da Silva. et al. Prevalência de infecção hospitalar pela bactéria do gênero klebsiella em uma Unidade de Terapia Intensiva. Revista Sociedade Brasileira de Clínica Médica. Juíz de Fora, v. 13, n. 2, p. 119-23, abr-jun, 2015.

PUGGINA, Ana Claudia. et al. Percepção da comunicação, satisfação e necessidades dos familiares em Unidade de Terapia Intensiva. Escola Anna Nery. Rio de Janeiro, v. 18, n. 2, Abr./Jun, 2014.

R Core Team. R: A language and environment for estatistical computing. R Foundation for Statistical Computing. Vienna, Áustria, 2017. Disponível em: http://www.R-project.org/.

SIEGEL, Sidney; CASTELLAN JR, N. John. Estatística não paramétrica para ciências do comportamento. Artmed Editora, 1975.

SINÉSIO, Marcia Cardoso Teixeira. et al. Fatores de risco às infecções relacionadas à assistência em unidades de terapia intensiva. Revista Cogitare Enfermagem. Brasília, v. 2, n. 23, p. 1-10, 2018.

SOUSA, Álvaro Francisco Lopes de. et al. Óbitos em idosos com infecção adquirida em Unidades de Terapia Intensiva. Rev. Bras. Enferm. Brasília, v. 70, n. 4, jul-ago, 2017.

THOMPSON, Mary Lou; MYERS, J. E.; KRIEBEL, D. Prevalence odds ratio or prevalence ratio in the analysis of cross sectional data: what is to be done?. Occup Environ Med, v. 55, p. 272$277,1998$.

World health Organization. Antimicrobial resistance. Draft global action plan on antimicrobial resistance. Report by the Secretariat. Executive Board. 136 th session. Provisional agenda item 8.1. 12 dezember 2014. 